\title{
Traveling waves in the Baer and Rinzel model of spine studded dendritic tissue
}

\author{
G J Lord \\ Department of Mathematics, Heriot-Watt University, Edinburgh, EH14 4AS, UK. \\ S Coombes \\ Nonlinear and Complex Systems Group, Department of Mathematical Sciences, \\ Loughborough University, Loughborough, Leicestershire, LE11 3TU, UK.
}

\begin{abstract}
The Baer and Rinzel model of dendritic spines uniformly distributed along a dendritic cable is shown to admit a variety of regular traveling wave solutions including solitary pulses, multiple pulses and periodic waves. We investigate numerically the speed of these waves and their propagation failure as functions of the system parameters by numerical continuation. Multiple pulse waves are shown to occur close to the primary pulse, except in certain exceptional regions of parameter space, which we identify. The propagation failure of solitary and multiple pulse waves is shown to be associated with the destruction of a saddle-node bifurcation of periodic orbits. The system also supports many types of irregular wave trains. These include waves which may be regarded as connections to periodics and bursting patterns in which pulses can cluster together in well-defined packets. The behavior and properties of both these irregular spike-trains is explained within a kinematic framework that is based on the times of wave pulses. The dispersion curve for periodic waves is important for such a description and is obtained in a straightforward manner using the numerical scheme developed for the study of the speed of a periodic wave. Stability of periodic waves within the kinematic theory is given in terms of the derivative of the dispersion curve and provides a weak form of stability that may be applied to solutions of the traveling wave equations. The kinematic theory correctly predicts the conditions for period doubling bifurcations and the generation of bursting
\end{abstract}


states. Moreover, it also accurately describes the shape and speed of the traveling front that connects waves with two different periods.

Key words: neural traveling waves, dendritic spines, connections to periodics, kinematic formalism 


\section{Introduction}

As dendrites form the predominant elements in neurons, so dendritic spines form the dominant component of many types of dendritic trees. They are small mushroom like appendages with a bulbous head (with surface area of order $1 \mu \mathrm{m}^{2}$ ) and a tenuous stem (of length around $1 \mu \mathrm{m}$ ) and may be found in their hundreds of thousands on the dendritic tree of a single cortical pyramidal cell. These extensions of the dendritic tree provide junction points for the axons of other neurons (i.e., provide surface area for synapses), and thus serve as loci for receiving inputs (especially of the excitatory kind). There is increasing evidence that dendritic spines may be important components in many kinds of neural microcircuits. In the human nervous system, dendritic spines are especially prominent in the cerebellar cortex, basal ganglia, and cerebral cortex. In the cerebral cortex approximately 80 percent of all excitatory synapses are made onto dendritic spines, whereas only around 30 percent of inhibitory synapses are made onto dendritic spines. Dendritic spines are thought to have several different functional roles that include subserving cognitive functions such as learning and memory [1,2,3], logical computations [4] and pattern matching tasks $[5,6]$.

Experimental methods have been developed that can measure the electrical events in dendrites directly and other, more recent, techniques have also allowed optical recordings from dendritic spines. These types of direct observations confirm earlier speculations that spine heads possess excitable channels capable of generating action potentials (see [7] for a discussion). As a consequence signal processing in the spatially extended dendritic tree is likely to be highly nonlinear, due to the excitable nature of spine heads. Theoretical explorations of the biophysical consequences of such nonlinearities have shown that global signals, in the form of traveling waves, may arise from a succession of local all-or-none events at the spine heads. Most of this theoretical work has been done using simulations of the biophysically realistic model of Baer and Rinzel [8] or with mathematical treatments of reduced versions of this model such as in the work by Chen [9], Zhou [10,11] and Coombes $[12,13]$. In this paper we present further results regarding traveling waves in 
the full Baer and Rinzel model using a mixture of computational analysis and a kinematic formalism. The combination of these two branches of mathematical analysis allows us to explore the conditions for the existence of traveling waves, previously observed with direct numerical simulations by Baer and Rinzel [8]. Moreover, these techniques lend themselves naturally to the construction of previously unobserved double and multi-pulse solutions. Periodic traveling waves are also found to co-exist alongside with these multi-pulse type solutions and, once again, the same set of numerical techniques may be used to construct the dispersion relation (i.e. wave speed as a function of period). The kinematic formulation treats the times of wave crests as fundamental and complements the numerical construction (and continuation) of traveling waves in that it provides a natural way in which to determine the stability (in some weak sense) of a periodic wave in terms of its dispersion relation. This kinematic stability condition is found to correctly determine the conditions for a period doubling bifurcation. Importantly, the kinematic formalism may be applied to the study of non-periodic waves. Numerically obtained dispersion curves are used within the kinematic framework to predict the existence of two types of irregular wave within the full Baer and Rinzel model. The first are obtained as exact solutions to the kinematic equations of motion and describe waves that (in the traveling frame) connect periodic waves of differing periods via some smooth transition layer. We adopt the term from dynamical systems and call these solutions heteroclinic connections between periodic orbits. The second may be regarded as arising through an instability, predicted from the kinematic theory, that gives rise to a wave in which the crests of a short wave train bunch together into packets which are separated by larger intervals, but which may themselves repeat regularly. We term these as bursting solutions.

In section 2 we introduce the Baer and Rinzel (BR) model which describes a uniform unbranched dendritic tree with a continuous distribution of active spines, and describe previously observed numerical behavior. Since the majority of numerical results for the BR model relate to traveling waves we introduce a traveling wave coordinate in section 3 and outline the procedures we use to construct solitary waves and multi-bump solutions (homoclinic connections to a fixed point) and periodic waves (possessing multiple periods). The fundamental parameters of the BR model are identified as the spine stem resistance 
$r$ and spine density $\rho$. A thorough exploration of the $(r, \rho)$ parameter space is used to illustrate the properties of such waves (most notably speed) and to determine the borders in parameter space defining propagation failure. A comparison of these various types of waves suggests that periodic orbits bifurcate from homoclinic orbits at a co-dimension one point whilst multi-pulse solutions branch from single pulse solutions at a co-dimension two point. In fact the BR model is found to support both a family of n-pulse solutions, denoted $H_{n}$, and a family of n-periodic orbits, denoted $P_{n}$, all of which are found to branch from the single pulse solution. In the $(r, \rho)$ parameter plane the regular traveling wave solutions that branch from the single are all found to lie close to the primary $H_{1}$ orbit. In section 4 we develop a kinematic theory of non-periodic waves based around the dispersion curves obtained in section 3 and derive the conditions for stability of waves in this framework. Period doubling bifurcations are shown to be associated with the coalescence of a pair of stable and unstable waves of so-called super-normal speed. The kinematic theory predicts the existence of connections to periodics, recently observed numerically by Coombes [13] and previously unobserved bursting solutions. The transition layer of a connection is shown to have a universal shape with a group velocity that is a function of the periods of the wave at large distances from the transition layer. Bursting solutions are predicted to occur in the neighborhood of an unstable wave of supernormal speed. Direct numerical simulation is used to show the success of the kinematic approximation in describing these types of irregular waves. Finally in section 5 we summarize the results of our analysis and discuss extensions of this work.

\section{The biophysical model}

In order to analyze the interactions between spines Baer and Rinzel formulated a new type of cable theory for dendritic systems which has a continuous distribution of excitable spines [8]. The formulation retains the notion that there is no direct electrical coupling between neighboring spines. Voltage spread along a uniform passive cable is the only way for the spines to interact; spines are electrically independent from one another. The active membrane in the 
spine-head is modeled by Hodgkin-Huxley kinetics. The continuum model of Baer and Rinzel with constant spine density $\rho$ is written with the following equations:

$$
\begin{aligned}
& C \frac{\partial V}{\partial t}=-g_{L}\left(V-V_{L}\right)+\frac{1}{r_{a} \pi d} \frac{\partial^{2} V}{\partial x^{2}}+\rho \frac{\widehat{V}-V}{r} \\
& \widehat{C} \frac{\partial \widehat{V}}{\partial t}=-I(\widehat{V}, m, n, h)-\frac{\widehat{V}-V}{r} .
\end{aligned}
$$

Equation (1) describes the dynamics of a uniform passive cable (which we take to be of infinite extent) of diameter $d$ with voltage $V(x, t)$ such that the last term on the right hand side is proportional to the flow of current between the cable shaft and the spine-head above the shaft via an ohmic spine-stem resistance of strength $r$. The terms $V_{L}$ and $g_{L}$ are respectively a constant leakage reversal potential and leakage conductance (or inverse leakage decay constant). The parameter $r_{a}$ represents the intracellular resistance per unit length of the cable. The electronic length constant $\lambda$ is given by $\lambda^{2}=$ $1 /\left(\pi d r_{a} g_{L}\right)$ and the membrane time constant (of the dendritic cable) by $\tau=$ $C / g_{L}$. (Note that in the original paper of Baer and Rinzel the spine density is defined in terms of unit length. To convert to the notation of this original paper simply write $\rho=\bar{n}\left(R_{\infty} / R_{m}\right)$, where $\bar{n}$ is the spine density per unit electronic length, $R_{\infty}$ is the input resistance if the branch were semi-infinite and $R_{m}$ is the resistance across a unit area of passive membrane. Equivalently one may write $\left.\rho=\bar{n}\left(\lambda r_{a} g_{L}\right)\right)$. The excitable dynamics of the voltage in the spine-head $\widehat{V}(x, t)$ is driven by the flow of current from the shaft to the spine and is described with equation (2). The function $I$ in (2) is used to denote the Hodgkin-Huxley dynamics. In the Hodgkin-Huxley model of excitable nerve tissue the membrane current arises mainly through the conduction of sodium and potassium ions through voltage dependent channels in the membrane. The contribution from other ionic currents is assumed to obey Ohm's law. In fact the Hodgkin-Huxley dynamics is considered to be a function of $\widehat{V}$ and three time and voltage dependent conductance variables $m, n$ and $h$ :

$$
I(\widehat{V}, m, n, h)=g_{K} n^{4}\left(\widehat{V}-V_{K}\right)+g_{N a} h m^{3}\left(\widehat{V}-V_{N a}\right)+g_{L}\left(\widehat{V}-V_{L}\right),
$$

where $g_{K}, g_{N a}$ and $g_{L}$ are constants and $V_{L}, V_{K}$ and $V_{N a}$ represent the constant membrane reversal potentials associated with the leakage, potassium 
and sodium channels respectively. For simplicity we have considered the currents due to the leakage terms in the model of the cable and those in the model of the spine-head to be identical. The conductance variables $m, n$ and $h$ take values between 0 and 1 and approach the asymptotic values $m_{\infty}(\widehat{V})$, $n_{\infty}(\widehat{V})$ and $h_{\infty}(\widehat{V})$ with time constants $\tau_{m}(\widehat{V}), \tau_{n}(\widehat{V})$ and $\tau_{h}(\widehat{V})$ respectively. Summarizing, we have that

$$
\tau_{X}(\widehat{V}) \frac{\partial X}{\partial t}=X_{\infty}(\widehat{V})-X, \quad X \in\{m, n, h\}
$$

The six functions $\tau_{X}(\widehat{V})$ and $X_{\infty}(\widehat{V}), X \in\{m, n, h\}$, are obtained from fits with experimental data [14] (given in appendix A). From now on we choose capacitances $C$ and $\widehat{C}$ in (1) and (2) such that $C=\widehat{C}$ and take $C=1$ without loss of generality. It is also convenient to choose a length scale such that $r_{a} \pi d=1$.

Baer and Rinzel have shown that the combination of diffusion along the cable combined with input from electronically separated active spines can lead to the propagation of a traveling wave. In essence a brief external input, say in the form of a synaptic current, leads to a spread of potential along the cable. The dynamics in the spine head is driven by this potential and for sufficiently large drive an action potential may be generated. There is then a large reinjection of current back into the dendrite that causes a further spread of potential along the cable so that the process is self-perpetuating. Thus one expects firing activity in the spines to ride the crests of a succession of diffusing pulses in the dendritic cable. Simulations show that the speed of this traveling wave decreases as a function of the spine density and there is propagation failure for too large a spine-stem resistance or too small a spine density.

An exact mathematical treatment of the BR model is yet to be performed, due in part to its complexity. By replacing the model spine head dynamics with the FitzHugh-Nagumo system and further ignoring the dynamics of the refractory variable precise statements about wave initiation [9] and traveling wave fronts have been made [11]. The inclusion of dynamics describing the refractory process complicates any analysis. In order to make mathematical progress Coombes and Bressloff [12] formulated a reduction of the BR model, the so-called spike-diffuse-spike model, which retains a description of refrac- 
toriness. In this model the excitable spine head dynamics is described with a simple integrate-and-fire process rather than the full Hodgkin-Huxley dynamics. By using a crude representation for the shape of an action potential this has allowed the analytical construction of both traveling wave solutions and dispersion curves [13]. In the next section we focus on the numerical construction of traveling waves in the full BR model and show that it supports many of the types of waves suggested by direct numerical simulations and the analysis of similar models with reduced descriptions of the excitable spine head dynamics. Importantly, this numerical approach allows the study of multipulse solutions and is useful in helping to organize the bifurcation structure of regular traveling waves. Moreover, we are able to numerically calculate the dispersion curves for periodic waves and to use these to predict the existence of waves of so called supernormal speed.

\section{Traveling wave frame}

To construct traveling wave solutions in the BR model we assume solutions of the form $V(x, t)=V(\xi)$ where $\xi=c t-x$ and similarly for $\widehat{V}, m, n$ and $h$ and look for bounded orbits. Once an initial solution has been constructed one may then numerically continue the speed of the wave in one or more system parameters. Traveling waves of speed $c$ are described by the following system of six coupled ordinary differential equations:

$$
\begin{aligned}
V_{\xi} & =W \\
W_{\xi} & =c W+\epsilon V-\frac{\rho}{r} \widehat{V}-g_{L} V_{L} \\
c \widehat{V}_{\xi} & =-\widehat{\epsilon} \widehat{V}+\frac{V}{r}-g_{K} n^{4}\left(\widehat{V}-V_{K}\right)-g_{N a} h m^{3}\left(\widehat{V}-V_{N a}\right)+g_{L} V_{L} \\
c \tau_{X} X_{\xi} & =X_{\infty}(\widehat{V})-X, \quad X \in\{m, n, h\},
\end{aligned}
$$

where $\epsilon=g_{L}+\rho / r$ and $\widehat{\epsilon}=g_{L}+1 / r$. We solve the system of ODEs given by (5) as a boundary value problem and impose either periodic boundary conditions to compute periodic traveling waves or projection boundary conditions [15] to compute traveling pulse solutions.

The fixed point of this system is given by $(V, W, \widehat{V}, m, n, h)=\left(V^{*}, 0, \widehat{V}^{*}, m^{*}, n^{*}, h^{*}\right)$ 
where $X^{*}=X_{\infty}\left(\widehat{V}^{*}\right), X \in\{m, n, h\}, V^{*}=\left(\rho \widehat{V}^{*} / r+g_{L} V_{L}\right) / \epsilon$ and $\widehat{V}^{*}$ satisfies $\widehat{V}^{*}=G\left(\widehat{V}^{*}\right)$ with

$$
G(x)=\frac{g_{K} n_{\infty}^{4}(x) V_{K}+g_{N a} h_{\infty}(x) m_{\infty}^{3}(x) V_{N a}+g_{L} V_{L}(1+1 /(\epsilon r))}{\widehat{\epsilon}+g_{K} n_{\infty}^{4}(x)+g_{N a} h_{\infty}(x) m_{\infty}^{3}(x)-\rho /\left(\epsilon r^{2}\right)} .
$$

For parameter values considered in this paper we have found numerically that there is only one solution to $\widehat{V}^{*}=G\left(\widehat{V}^{*}\right)$. Since the fixed point equations $X_{\infty}\left(\widehat{V}^{*}\right)=X^{*}$ also have unique solutions (for $X \in[0,1]$ ) we expect that generically the traveling wave BR equations possess a single fixed point. Uniqueness of the fixed point excludes the possibility of bifurcations involving multiple fixed points such as global heteroclinic bifurcations. Numerical construction of the eigenvalues of the Jacobian matrix of the traveling wave system (5) also shows that, for the considered parameter ranges, the fixed point has a five dimensional stable manifold with a single pair of complex conjugate eigenvalues and a one dimensional unstable manifold.

\subsection{Homoclinic orbits}

To construct homoclinic orbits in the BR model we look for bounded orbits that are homoclinic connections to the rest state in the traveling coordinate frame. We implemented in Auto97 [16] the projection boundary conditions of [15]. We truncate and rescale (5) to the interval $[0,1]$ and project out the 5 dimensional stable manifold at $\xi=0$, the 1 dimensional unstable manifold at $\xi=1$ and impose an integral phase condition. It is then possible to perform numerical continuation of solutions in any two of the system parameters such as wave-speed $c$, resistance $r$ or density $\rho$ (the homoclinic orbits exist in a co-dimension one subset of parameter space). To continue a limit point of homoclinic orbits we introduce a further free parameter and continue in $c, r$ and $\rho$. Projection boundary conditions are more accurate than a large fixed period periodic approximation since the error due to truncation to a finite interval is exponentially small $[15,17]$. Good initial guesses are required for Newton's method, involved in the solution to the boundary value problem, to converge. For the approximation of a single pulse homoclinic orbit we used a periodic orbit of large period. By following the period doubled solutions that 
arise from period doubling bifurcations out to large period it is also possible to obtain initial guesses for 2-pulse and 4-pulse homoclinics. In general it is also possible to compute an $n$-pulse homoclinic by pasting together multiple copies of the 1-pulse solution (or other available multi-pulse solutions). This technique was applied to find an initial guess for the 3-pulse solutions.

By continuing the speed of the solitary pulse as a function of the spine density $\rho$ and the spine stem resistance $r$ it is a simple matter to quantify the original observation of Baer and Rinzel [8] that propagation failure may result for too large a spine stem resistance and too small a spine density. In more detail, consider a fixed resistance of $r=0.05$ and examine the wave speed as the density varies (figure 1). We see from figure 1 (a) that the speed of the solitary wave decreases as the spine density increases. Figure 1 (b) magnifies the region around the turning point in figure 1 (a), from which we see that the multipulse solutions exist for lower densities than the 1-pulse homoclinic $H_{1}$. This figure clearly shows the co-existence of multiple forms of homoclinic orbits at a given parameter value. Note that, for clarity, we have not plotted in figure 1 (a) and (b) all curves of $H_{2}, H_{3}$ and $H_{4}$ solutions. In figure 1 (c) and (d) we have plotted a more complete picture for the $\mathrm{H}_{2}$ curve of solutions. Note there are two limit points shown in the blow up in (d) indicating that the secondary branches of homoclinics do not always co-exist alongside the main branch (seen in figure 1 (a)).

In common with many other models of excitable nerve tissue we find that the $n$-pulse orbits may be categorized into slow and fast solutions. In figure 2 we show some examples of $n$-pulse solutions to the traveling wave equations of the full BR model with $r=0.05$ and $\rho=25$. Figure 2 (a) shows the fast and slow 1-pulse homoclinic orbit that exist at the same parameter values. For the multi-pulse orbits the situation is similar except there are sets of fast and slow waves that differ in the separation between peaks (and so the time spent in the neighborhood of the origin). This can be seen in figure 2 (b), (c) and (d) for the 2, 3 and 4-pulse homoclinics where we have split the plots into fast and slow waves (for clarity only one of each type of wave is plotted for the 4-pulse homoclinic). 
(a)

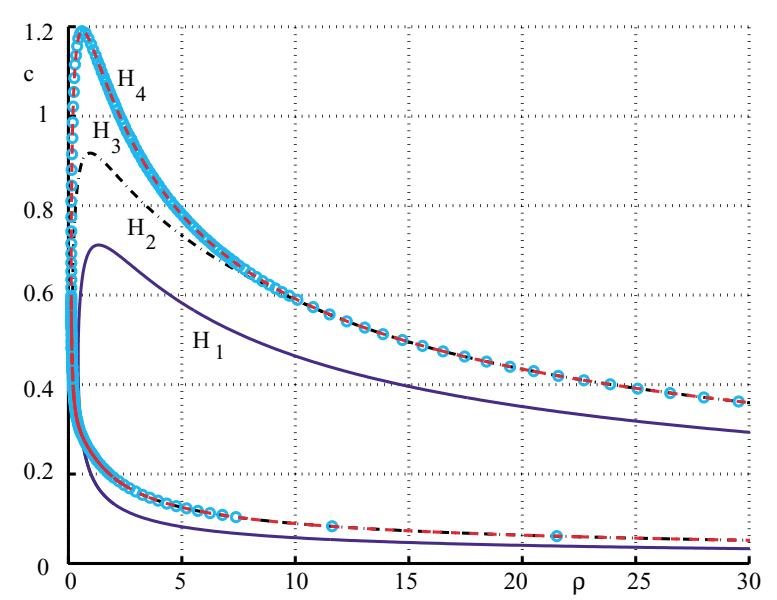

(c)

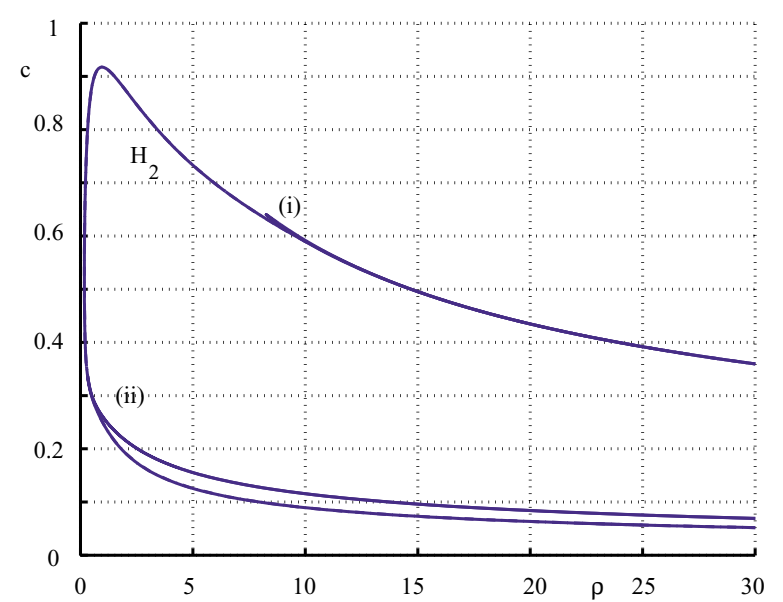

(b)

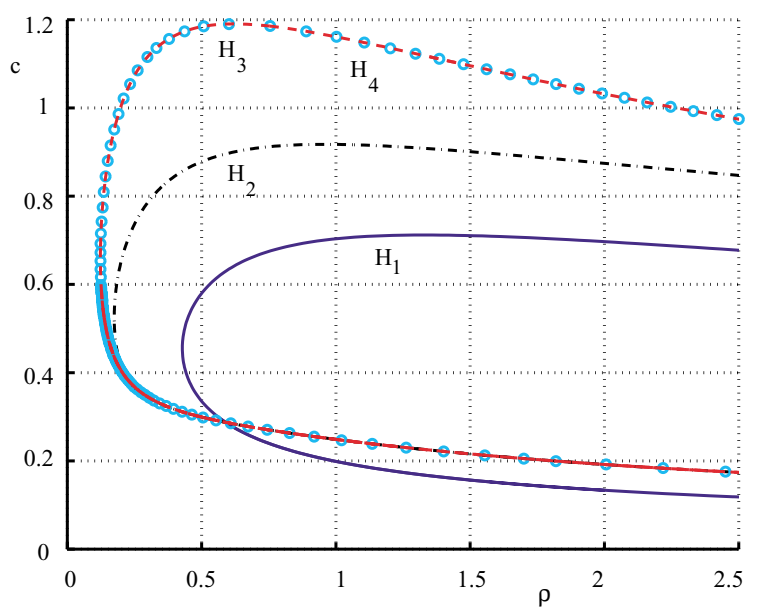

(d)

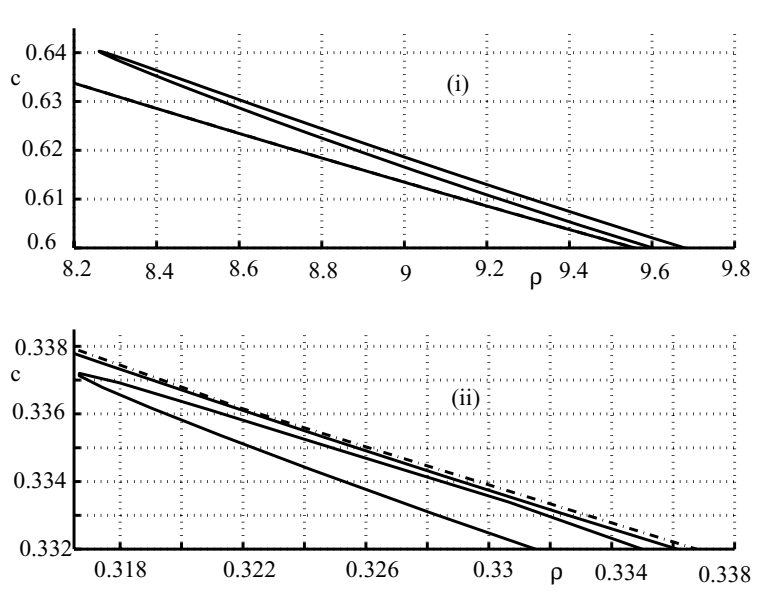

Fig. 1. (a) The speed of homoclinic orbits $H_{1}, H_{2}, H_{3}$ and $H_{4}$ for $r=0.05$ as a function of the spine density $\rho$. (b) Blow-up of (a) showing that, that for a fixed (small) $r$, multi-pulse solutions can exist at smaller values of $\rho$ than for $H_{1}$. Note that, for clarity, only the main solution branches have been plotted for $H_{2}, H_{3}$ and $H_{4}$. (c) Main and secondary branches of $H_{2}$ solutions. (d) Blow ups of (c) showing that the secondary branches occupy smaller windows of parameter space than the main branch.

In figure 3 we plot the homoclinic solutions from figure 2 at a larger value of the spine-stem resistance, $r=1$. Figure 3 (a) is the partner to figure 2 (a), and likewise for (b), (c) and (d). By comparing figures 2 and 3 it is apparent that multi-bump solutions have well separated peaks for large $r$, whilst for smaller $r$ the peaks can run into one another. 
(a)
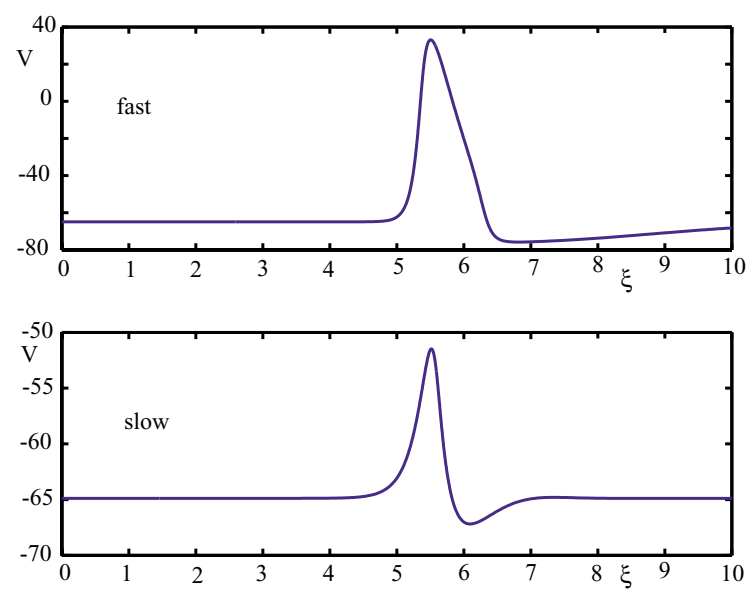

(c)
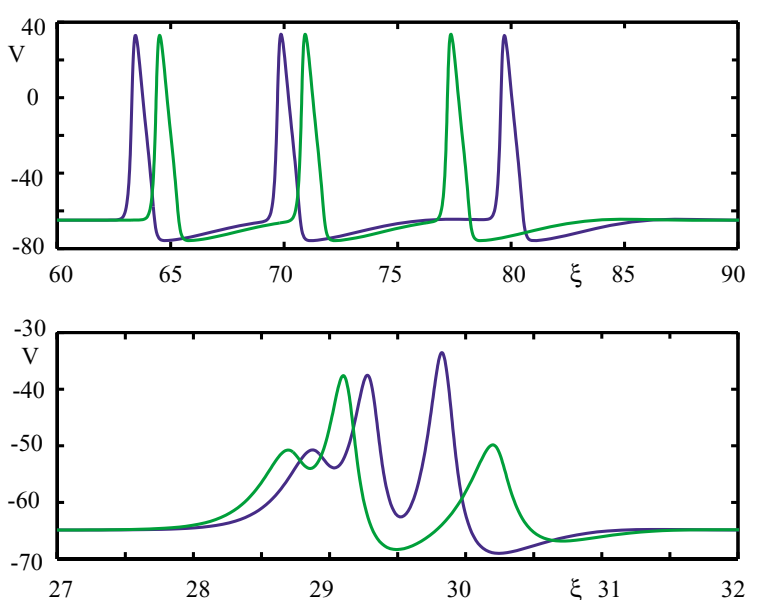

(b)
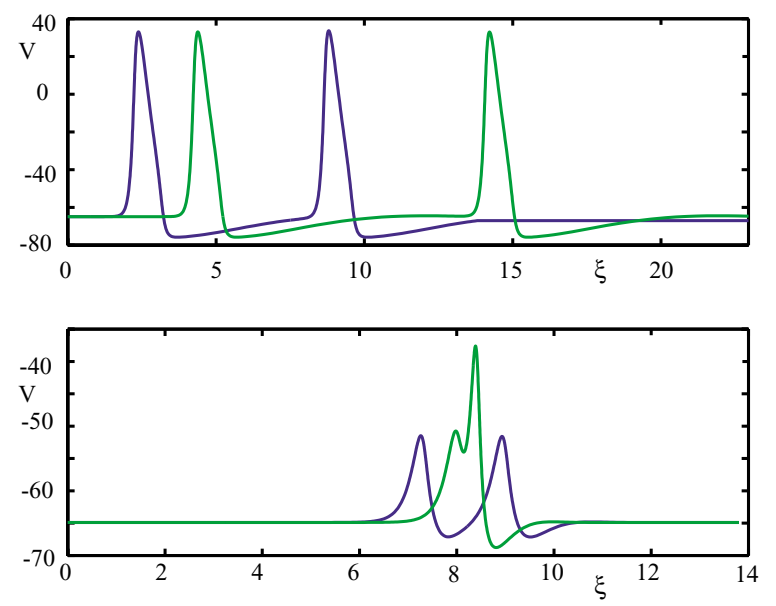

(d)
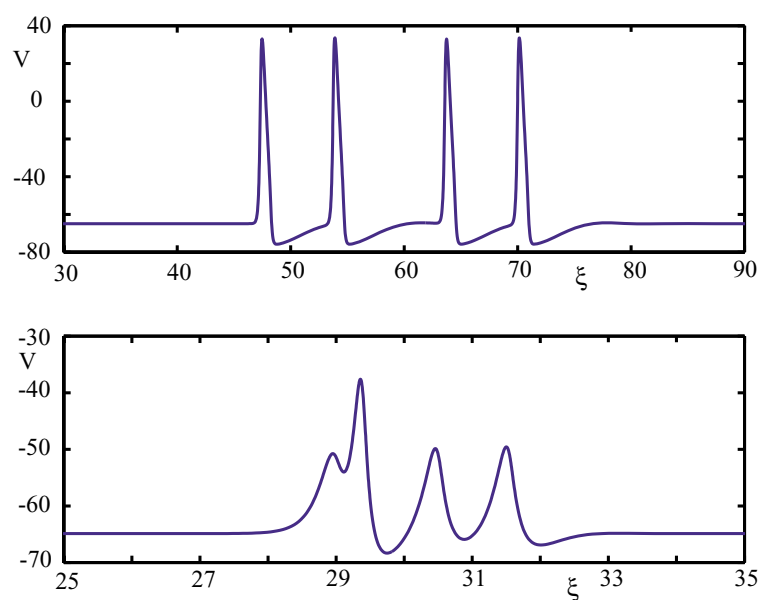

Fig. 2. Examples of traveling pulse solutions in the BR model obtained as homoclinic connections to the fixed point for $r=0.05$ and $\rho=25$. In each of (a) to (d) the waves in the upper window have speeds greater than those in the window below. (a) $H_{1}$. (b) $H_{2}$. (c) $H_{3}$. (d) $H_{4}$.

For both fast and slow waves, the overshoot of a homoclinic connection around the saddle-focus is typically heavily damped so that in numerical simulations it can be hard to make out more than one maxima in the oscillatory tails of orbits such as those in figures 2 and 3. We suggest later, using a weak notion of stability, that for waves on a common main branch it is the faster of the two branches that is in some sense stable. Note that wave speed can be read off from figure 4 
(a)
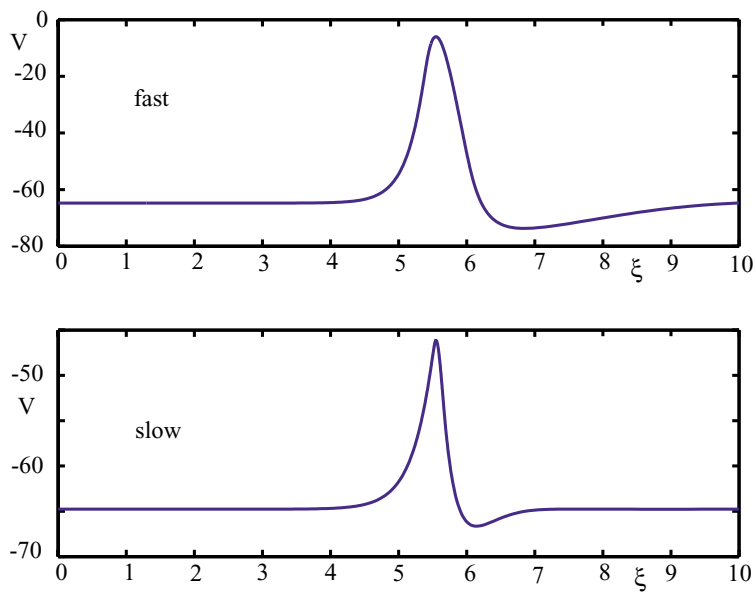

(c)
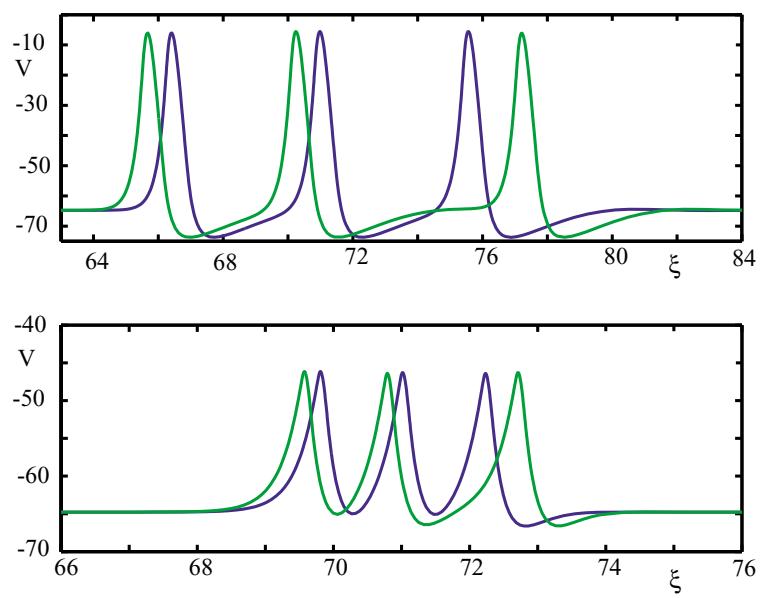

(b)
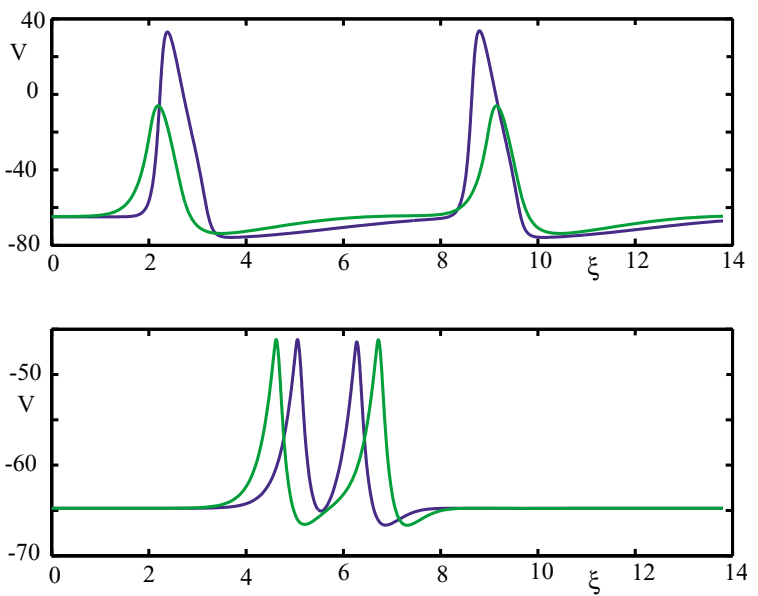

(d)
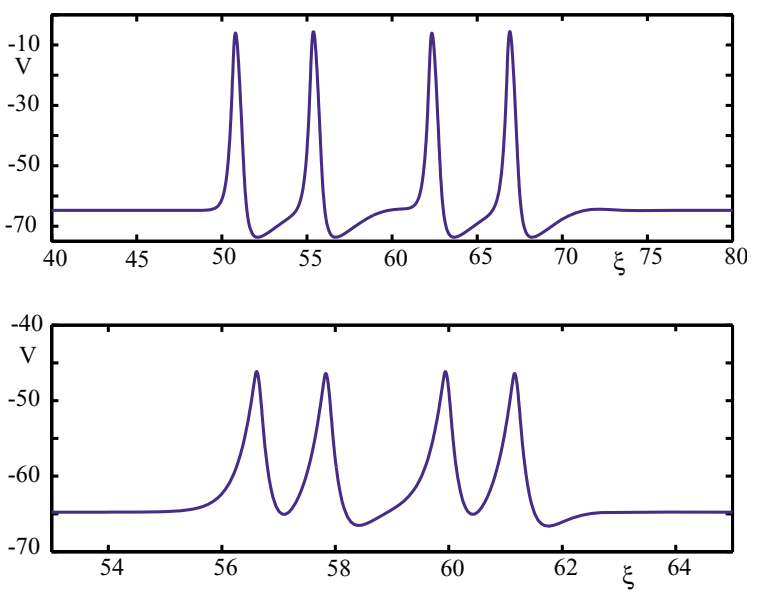

Fig. 3. Examples of traveling pulse solutions in the BR model obtained as homoclinic connections to the fixed point, for a larger value of the spine stem resistance than in figure 2, namely $r=1$. (a) $H_{1}$. Upper (lower) window shows fast (slow) solution (b) $H_{2}$. Upper (lower) window shows two fast (slow) solutions. (c) $H_{3}$. Upper (lower) window shows two fast (slow) solutions. (d) $H_{4}$. Upper (lower) window shows one fast (slow) solution. In all cases the density is given by $\rho=25$.

In figure 4 (a) we show the results of continuation of the 1-pulse homoclinic in resistance $r$ and wave speed $c$ (solid line) for a fixed density $\rho=25$. Included on this plot is the saddle node of periodic orbits $S N_{1}$ (the point where a fast and slow periodic orbit coalesce, further discussed in section 3.2). In figure 4 (b) we have plotted the 2, 3 and 4 pulse homoclinics on a common window of parameter space. These have been plotted separately to allow the reader 
(a)

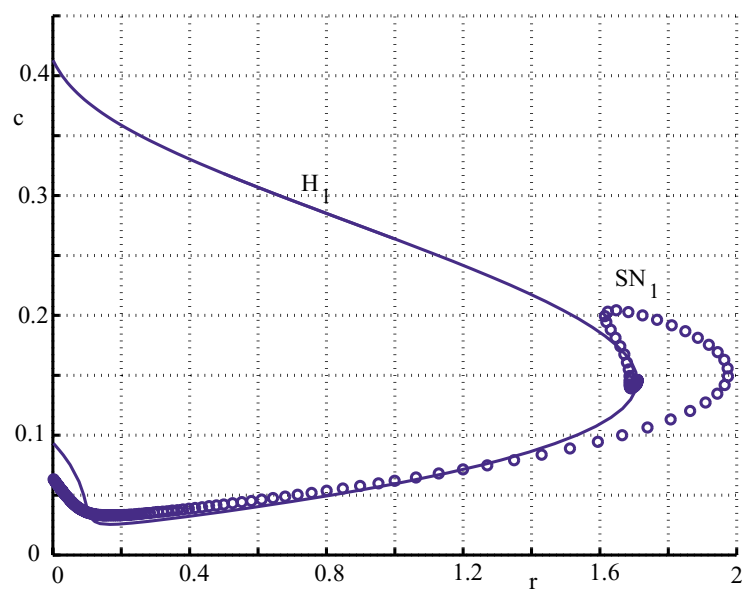

(b)
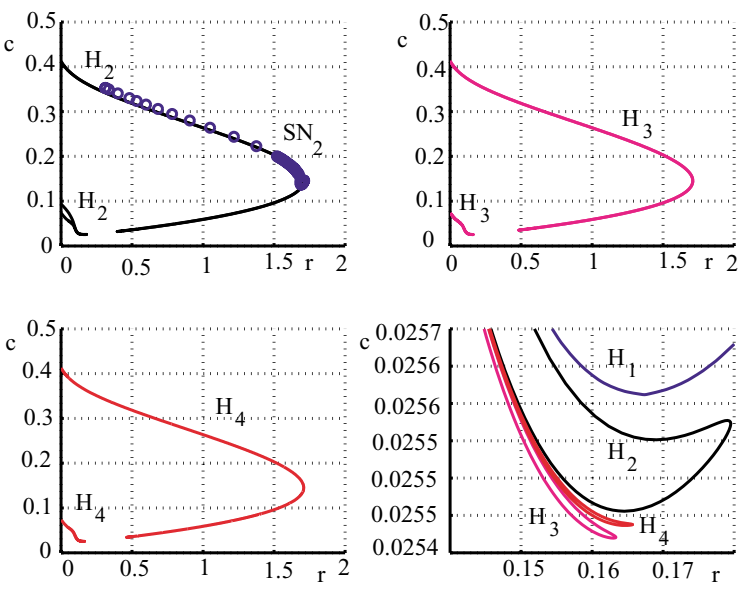

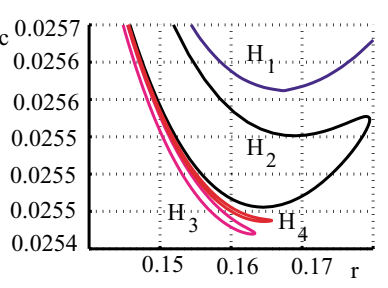

Fig. 4. In (a) we show the speed of the solitary wave $H_{1}$ as a function of the spine-stem resistance $r$ for $\rho=25$. The open circles denote the point where the stable and unstable $P_{1}$ orbits coalesce, labeled $S N_{1}$. In (b) we have plotted $H_{2}-H_{4}$ in the same region of parameter space. $S N_{2}$ denotes the point where a stable and unstable $P_{2}$ orbit coalesce. Note the existence of gaps for $H_{2}, H_{3}$ and $H_{4}$, where solutions cease to exist. In the final of these four figures we show a blowup this small $r$ phenomenon (on the side of the gap with smallest $r$ ).

to distinguish the individual curves. We find that slow multi-pulse solutions cease to exist in a small region of parameter space (roughly $0.2<r<0.4$ ) and that there are disconnected branches of $H_{n}$ solutions for $n>1$. The $H_{1}$ solution branch is, in contrast, connected. We include a blow up around $r=0.2$ to show that the multi-pulse solutions have a fine structure of two branches that coalesce at a limit point. A similar picture is obtained on the right hand side of the multi-pulse gap at around $r=0.4$. Hence, one might expect the creation of a stable/unstable pair of $H_{n}$ solutions $(n>1)$ at limit points in the $(r, \rho)$ plane. The observation of gaps in parameter space for multi-bump homoclinic orbits is unusual and would not seem to have a counterpart in the Hodgkin-Huxley model with pure diffusive coupling. It is already known that if a primary pulse converges to a saddle-focus (for semilinear parabolic equations on the real line), that, under suitable conditions, infinitely many $H_{n}$ solutions may bifurcate and among them are infinitely many stable ones [18]. This raises the interesting technical question of how to assess the stability of non-primary homoclinic solutions that emerge from the boundaries of a gap. 
From the structure of the speed curves $c=c(r)$ and $c=c(\rho)$ given in figures 4 and 1 it can be seen that the region of parameter space where $n$-pulse solutions fail to exist may be constructed by following the locus of points that define either of the limit points where $\mathrm{d} r / \mathrm{d} c=0$ or $\mathrm{d} \rho / \mathrm{d} c=0$ (where the fast and slow pair of homoclinic orbits coalesce). In fact at these points it would appear that a bifurcation to a periodic orbit can occur. We illustrate this in figure 4 by tracing out the locus of points where periodic $P_{1}$ and $P_{2}$ orbits cease to exist (see later). It can be seen that the point where a homoclinic orbit ceases to exist coincides with the appearance of a pair of (large period) periodics. In figure 5 (a) we plot the locus of $H_{1}, H_{2}, H_{3}$ and $H_{4}$ for fixed $c=0.1$ in the physiologically significant $(\rho, r)$ parameter plane. As in figure 4 we have plotted each branch separately for clarity. A blowup of figure 5 (a) is given in (b), where once again it is possible to see that multi-pulse solution branches are disconnected. In figure 5 (c) we plot the locus of points which define borders of existence for the solitary pulse (by continuation in $c$ of limit points in the $(\rho, r)$ plane). Note that for too high a spine-stem resistance or too low a spine density $\rho$ that the solitary pulse may fail to propagate. In figure 5 (d) we show the region in the $(\rho, r)$ plane, for small $r$, where multi-pulses $H_{1}, H_{2}, H_{3}, H_{4}$ can exist. Note that Homoclinic orbits $H_{2}, \ldots, H_{n}$ appear to bifurcate tangentially from $H_{1}$ in a co-dimension two bifurcation.

In Fig 6 we have brought together the information on the $H_{1}$ homoclinic into a 3D figure. We have added 3 parameter continuation of the limit points of $H_{1}$ and included the projections from figures 1, 4 and 5 .

\subsection{Periodic orbits}

Periodic orbits of the system of ODEs (5) correspond to periodic traveling waves, sometimes termed cnoidal waves. These are obtained by following branches of solutions that arise at Hopf bifurcation points in the ODE system. Once the initial periodic was located, period doubling bifurcations were detected automatically as numerical continuation was performed. Some examples of periodic traveling waves are shown in figure 7 . Homoclinic orbits are expected to arise, in a co-dimension one bifurcation, as the limit of periodic 
(a)
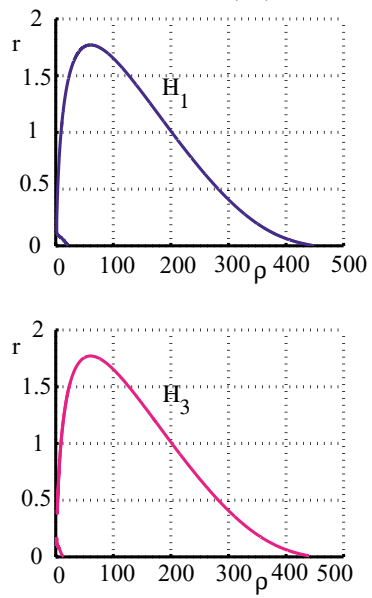

(c)
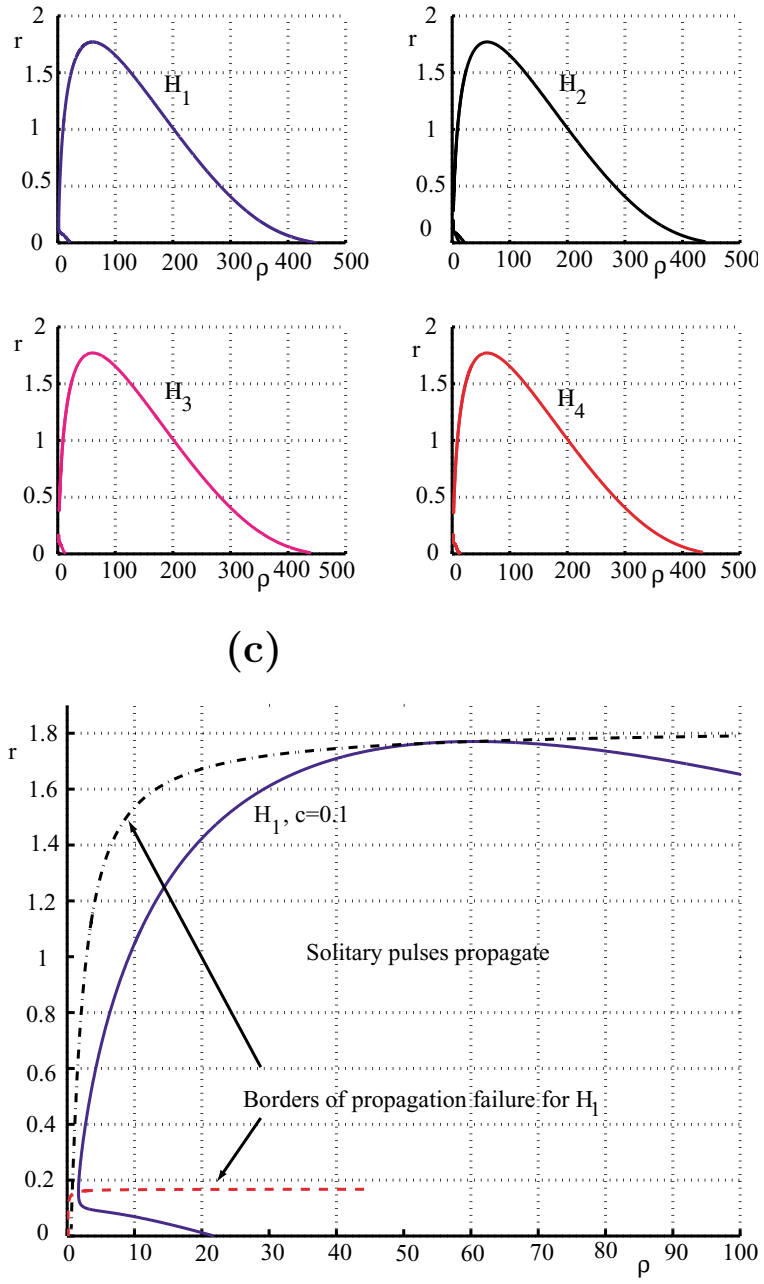

(b)
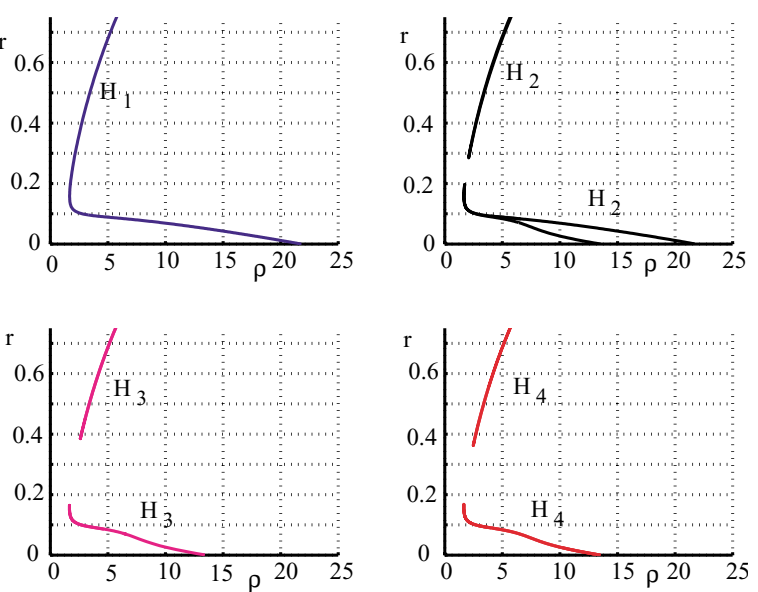

(d)

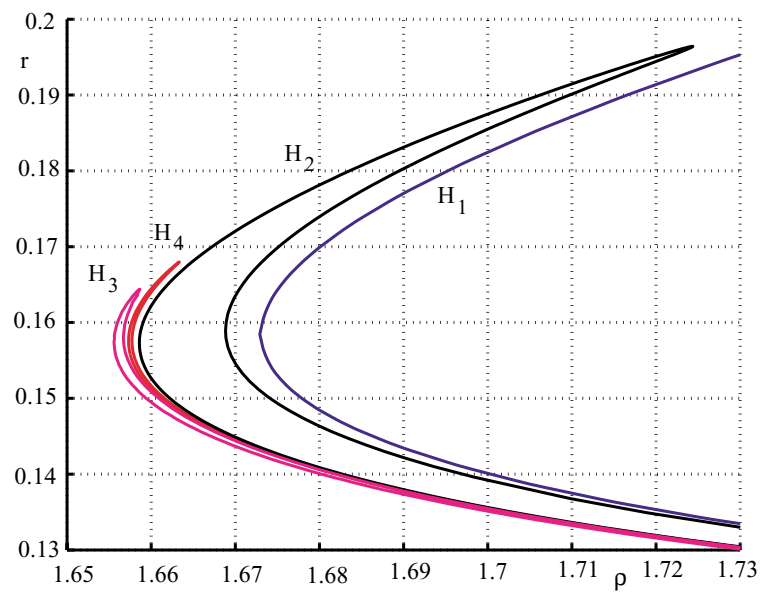

Fig. 5. Homoclinic orbits in the physiologically important $(r, \rho)$ parameter space. (a) $H_{1}-H_{4}$ for fixed wave speed $c=0.1$. (b) Detail for small density $\rho$. (c) $H_{1}$ branch with $c=0.1$ and continuation of limit points (varying $c$ ) delimiting borders of existence. (d) Co-existence of $H_{1}, H_{2}, H_{3}$ and $H_{4}$ at small $r$.

orbits when the period tends to infinity.

The situation for the periodic solutions is similar to that for the homoclinics in that periodic waves can come in fast/slow pairs, of which we predict that it is the faster of the two that is stable (see section 4). Unlike homoclinic solutions, the periodic orbits can exist for a range of periods $\Delta$, where $v(\xi+\Delta)=v(\xi)$ (and similarly for $\widehat{V}(\xi), m(\xi), n(\xi), h(\xi)$ ). For excitable nerve equations it is common to express properties of periodic orbits in terms of the dispersion curve $c=c(\Delta)$. This is easily calculated within the numerical framework 


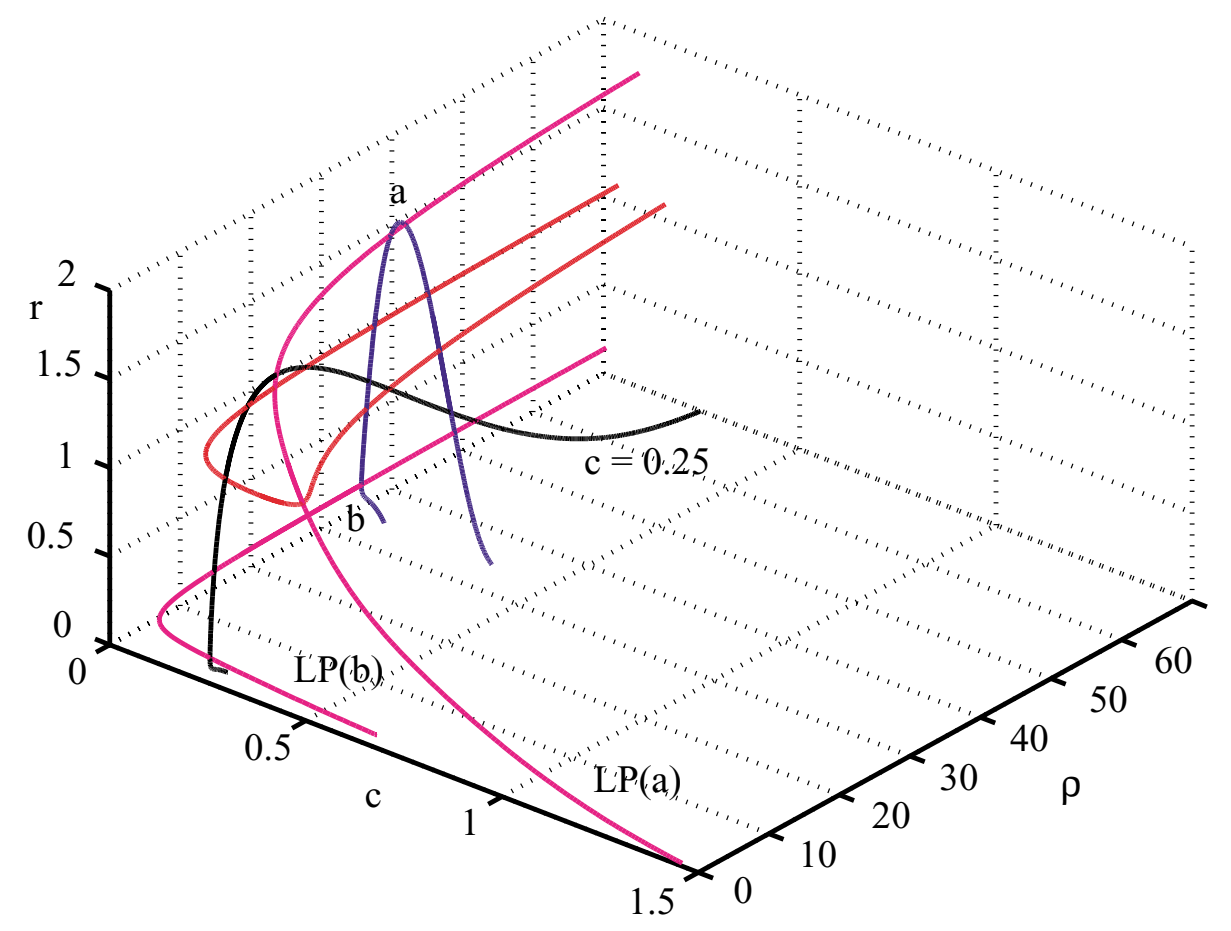

Fig. 6. Existence of $H_{1}$ in 3D parameter space. LP(a) and LP(b) denote the continuation of the limit points labeled by a and $\mathrm{b}$.

presented here by treating the unknown period $\Delta$ as a bifurcation parameter. Examples of dispersion curves for $P_{1}$ and $P_{2}$ are given in figure 8. It would seem that periodic orbits can fail to exist at a bifurcation point where fast/slow pairs of solutions coalesce at the limit point at minimum speed. For fixed parameters this typically happens for small periods. This is expected on physical grounds since the Hodgkin-Huxley dynamics of the excitable spine-head possesses a natural refractory period such that no action-potentials can be elicited for a certain time after a spiking event. Hence, the period at which propagation failure of periodics occurs is determined largely by the refractory period of the spine-head. For large periods we expect the speed of $P_{n}$ to approximate the speed of $H_{n}$.

Focusing on the faster (stable) branches of the dispersion curves of figure 8 we see that for large periods the speed of the wave is approximately that of the associated homoclinic orbit. However, as $\Delta$ decreases the speed can actually increase, resulting in a wave of so-called supernormal speed [19]. As $\Delta$ is 

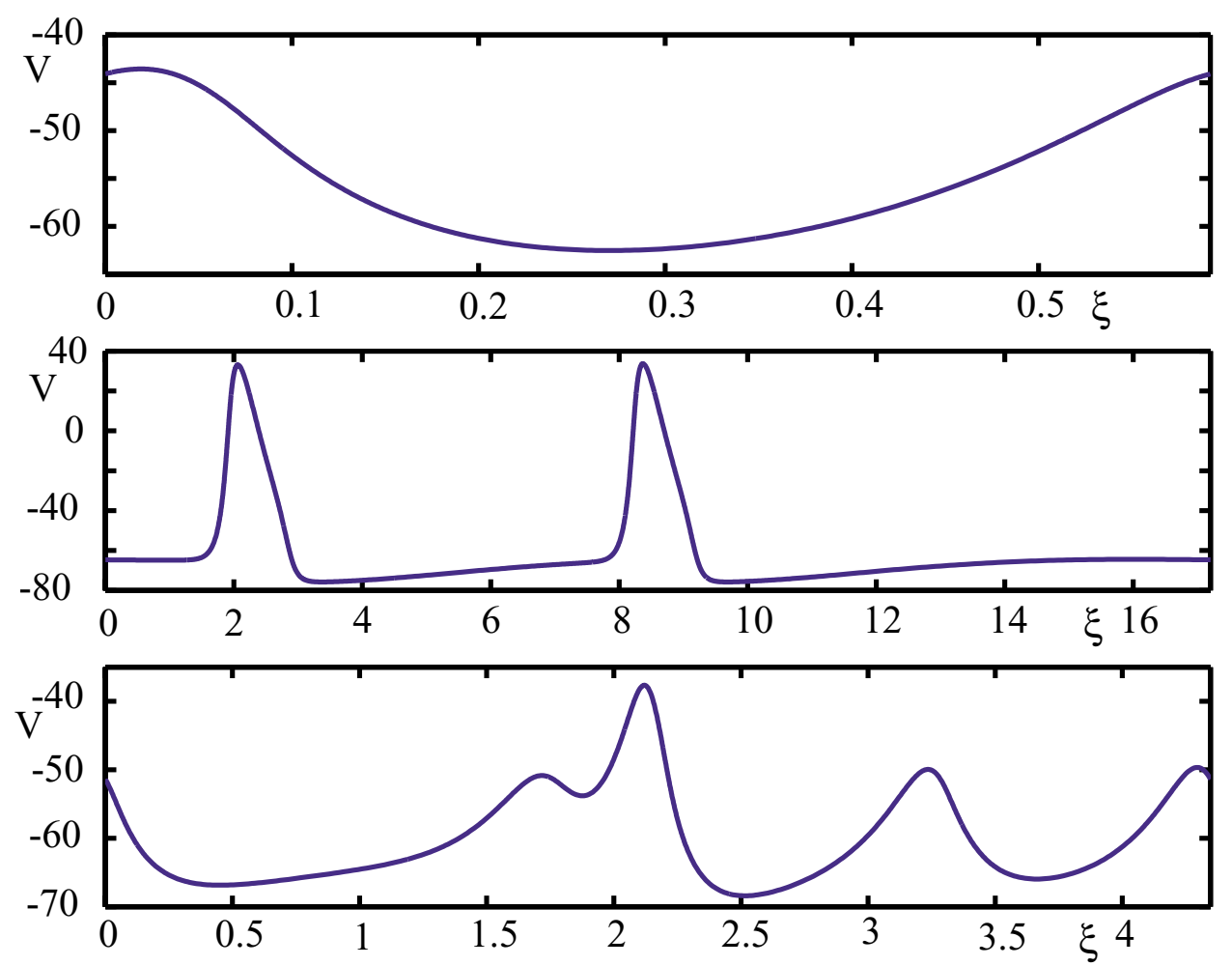

Fig. 7. Examples of periodic traveling waves in the BR model, with one, two and four fundamental periods ( $P_{1}, P_{2}$ and $P_{4}$ respectively). The two and four period solutions are obtained by following orbits that arise from period doubling bifurcations of the regular traveling periodic wave. Parameters are $\rho=25, r=0.05$.

decreased further the speed of the wave drops off before finally failing at the limit point where $\mathrm{d} \Delta / \mathrm{d} c=0$. At this point it joins with the slower wave which persists with increasing $\Delta$. Note that on the slower branch it is also possible to see waves of subnormal speed (with respect to the speed of the unstable homoclinic). The dispersion curve for $P_{1}$ will play a key role in the kinematic formalism developed later in section 4 . In figure 8 (b) we plot the dispersion curve for $P_{2}$ orbits of period $\Delta$ combined with the dispersion curve for $P_{1}$ orbits of period $\Delta / 2$. We find that the $P_{2}$ orbits cease to exist at stationary points on the $P_{1}$ dispersion curve, as illustrated in figures 8 (c) and (d). Interestingly the kinematic theory predicts a change in stability of $P_{1}$ orbits at just such points, suggesting that a $P_{1}$ orbit of supernormal speed can undergo a period doubling bifurcation to a $P_{2}$ orbit. A similar observation holds for waves of subnormal speed. Hence, we may associate period doubling bifurcations with stationary 
(a)

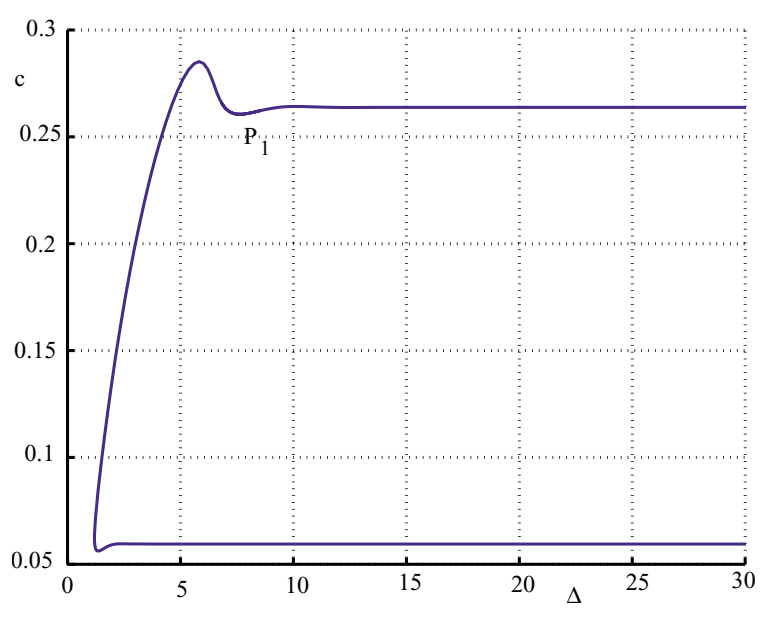

(c)

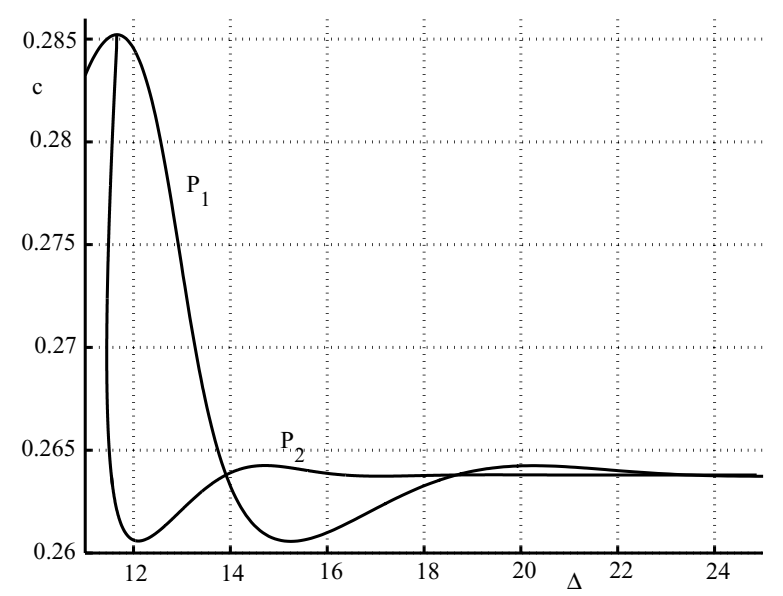

(b)

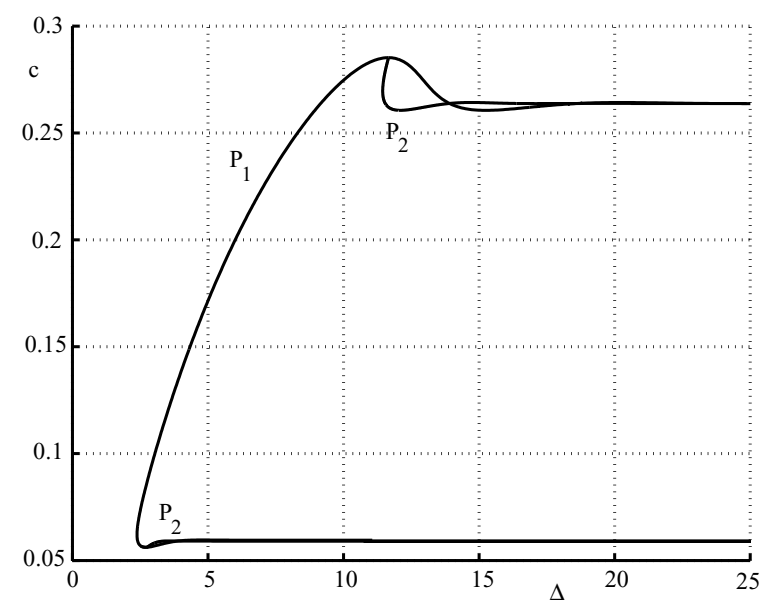

(d)

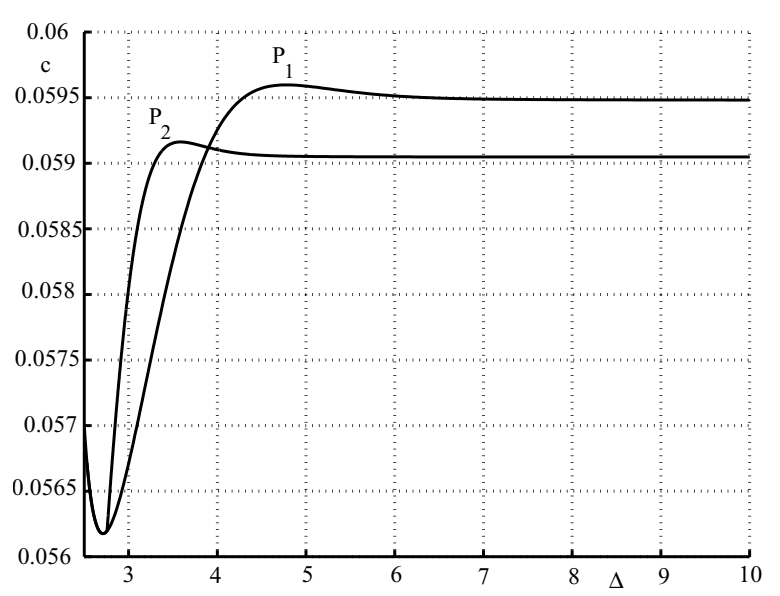

Fig. 8. Dispersion curves, $c=c(\Delta)$, for periodic waves with fixed $\rho=25$ and (a) $r=1$ for $P_{1}$ (b) dispersion curve for $P_{2}$ with dispersion curve for $P_{1}$ (with period $\Delta / 2$ superimposed). In (c) and (d) we produce blow-ups of (b) to illustrate that $P_{2}$ orbits cease to exist at stationary points of the $P_{1}$ dispersion curve.

points in the dispersion curve. It is therefore possible that bifurcations of waves of super and subnormal speed may lead to a period doubling cascade. A similar observation is expected to hold for other excitable systems coupled purely by diffusion.

In figure 9 we trace the locus of period doubling points in the $(\rho, r)$ parameter plane for a fixed value of the wave speed, $P D_{n}$, as well as the set of points defining propagation failure of $P_{1}$. We denote this latter border by $S N_{1}$ in 


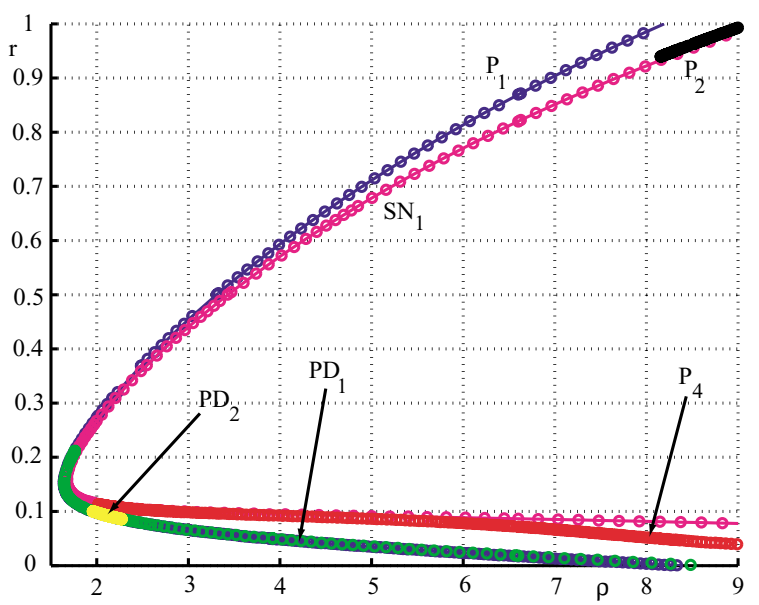

Fig. 9. The loci of points in the $(r, \rho)$ plane where the periodic waves $P_{1}, P_{2}$ and $P_{4}$ all have speed $c=0.1$. The line labelled $S N_{1}$ denotes the locus of points defining propagation failure of $P_{1} . P D_{1}$ and $P D_{2}$ denote the locus of period doubling bifurcations of $P_{1}$ and $P_{2}$ respectively.

loose analogy to a saddle-node bifurcation since it describes the coalescence of a stable and unstable periodic orbit. Referring back to figure 4 we find that tracing the locus of points where $\mathrm{d} \Delta / \mathrm{d} c=0$ ends at the point in the $(r, c)$ plane where homoclinic orbits cease to exist. As expected we find that at this point $\Delta \rightarrow \infty$. The propagation failure of multi-pulse solutions, $H_{n}$, is therefore associated with the loss of a saddle-node bifurcation $S N_{n}$. From figure 9 and more clearly from 10 (a blowup of figure 9) we see that the family of homoclinic orbits $H_{n}$ exists in a parameter window bordered by $S N_{1}$ and $P D_{1}$. Hence, homoclinic orbits may be found close to the primary periodic orbit $P_{1}$ in the $(\rho, r)$ parameter plane.

It would seem that the set of ordinary differential equations describing traveling waves in the full BR model allows a period-doubling cascade. Moreover, the orbit describing the primary regular periodic traveling may collide with the equilibrium point to create a homoclinic orbit. The same holds for each subsequent orbit in the period-doubling cascade. A system in which such a phenomenon exists must have at least two parameters, one controlling the period-doubling bifurcations and one controlling the period of the orbits. In parameter space, this corresponds to a sequence of period-doubling bifurcation curves, all ending in co-dimension two bifurcations involving homoclinic 


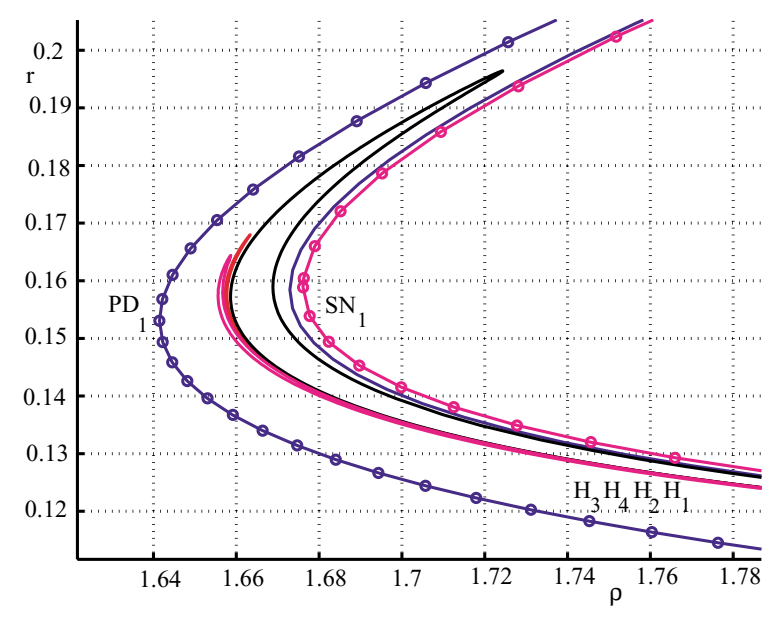

Fig. 10. A blowup of figure 9 including information about the existence of homoclinic orbits. The homoclinic orbits coexist with the primary periodic $P_{1}$, since they are sandwiched between $S N_{1}$ and $P D_{1}$.

orbits. Similar bifurcations are known to occur for excitable nerve equations whose fixed point (in the traveling wave frame) is unique and has real eigenvalues (see Glendinning [20] for a discussion). However, the fixed point in our study typically has a complex pair of eigenvalues and has more in common with nerve axon equations with a saddle focus in the the traveling wave frame. Since the equilibrium point is of the saddle focus type it is also likely that there exists a window of parameter space supporting horseshoe dynamics [21]. In terms of traveling wave dynamics we would therefore expect irregular (possibly chaotic) wave trains in this region of parameter space. The spatially extended FitzHugh-Nagumo equations with a piece-wise linear nonlinearity is one such example (where one can guarantee complex eigenvalues) and admits to mathematical analysis. For this model it has been possible to extend results due to Šilnikov and show the existence of an uncountable discrete family of traveling waves which are trains of infinitely many pulses [22]. Periodic and solitary waves in this model have also been extensively analyzed by Rinzel and Keller [23]. The study of more general nonlinearities in the FitzHugh-Nagumo model has also been possible, in some singular limit, and predicts a similar rich array of traveling wave solutions [24]. In the case that all the eigenvalues of the fixed point are real, Homoclinic-doubling can be caused by co-dimension two bifurcations involving a change in the orientation of the vector field around 
the homoclinic orbit: so called orbit flips and inclination flips [25] and is expected to be generic for traveling wave reductions of high dimensional nerve axon equations [20]. This interesting phenomenon would also be expected in the BR model if parameters of the Hodgkin-Huxley model were changed so as to guarantee real eigenvalues of the equilibrium point. In this case traveling pulses would not display any overshoot or oscillations in their tails.

\section{A kinematic analysis of spike train propagation}

Up until now we have focused on traveling waves that are either periodic or homoclinic to the fixed point (in the traveling wave frame). Since the underlying excitable dynamics of the spine-head is of Hodgkin-Huxley type these traveling waves have certain similarities with trains of action potentials. Hence, it is natural to identify the periodic wave with an infinite sequence of traveling pulses and homoclinic orbits as finite sets of traveling pulses. As for the Hodgkin-Huxley model of an axon, the BR model also supports action potentials that are irregularly spaced and that travel with different speeds (see Carpenter [24] for a discussion of irregular wave trains in nerve axon equations). A kinematic theory of wave propagation is one attempt to follow the progress of action potentials at the expense of a detailed description of the pulse shape [26]. Suppose that a pulse has a well defined arrival time at some position $x$ then we denote the arrival of the $n$th pulse at position $x$ by $T^{n}(x)$. A periodic wave, of period $\Delta$, is then completely specified by the set of ordinary differential equations

$$
\frac{\mathrm{d} T^{n}(x)}{\mathrm{d} x}=\frac{1}{c(\Delta)}
$$

with solution $T^{n}(x)=n \Delta+x / c$, where $c(\Delta)$ is the dispersion curve obtained previously by numerical means. The kinematic formalism assumes that there is a description of irregular spike trains in the above form such that

$$
\frac{\mathrm{d} T^{n}(x)}{\mathrm{d} x}=F\left(T^{n}(x), T^{n-1}(x), T^{n-2}(x), \ldots\right),
$$

which must reduce to (7) for periodic waves. Assuming that the most recent spike in the train has the strongest influence it is further assumed that $F\left(T^{n}(x), T^{n-1}(x), T^{n-2}(x), \ldots\right) \approx F\left(T^{n}(x), T^{n-1}(x)\right)$, which can only reduce 
to (7) if $F\left(T^{n}(x), T^{n-1}(x)\right)=F\left(T^{n}(x)-T^{n-1}(x)\right)$. The function $F(\Delta)$ is then chosen as $C^{-1}(\Delta)$, the reciprocal dispersion curve for the periodic wave. Hence, within the kinematic framework the dynamics of irregular traveling wave trains are described by (7) with the replacement $c(\Delta) \rightarrow c\left(T^{n}(x)-T^{n-1}(x)\right)$, where $T^{n}(x)-T^{n-1}(x)$ is recognized as the instantaneous period of the wave train at position $x$.

A steadily propagating wave train is stable if under the perturbation $T^{n}(x) \rightarrow$ $T^{n}(x)+u^{n}(x)$ the system converges to the unperturbed solution during propagation, or $u^{n}(x) \rightarrow 0$ as $x \rightarrow \infty$. For the case of uniformly propagating periodic traveling waves of period $\Delta$ we insert the perturbed solution in (7), so that to first order in the $u^{n}$

$$
\frac{\mathrm{d} u^{n}}{\mathrm{~d} x}=-\frac{c^{\prime}(\Delta)}{c^{2}(\Delta)}\left[u^{n}-u^{n-1}\right]
$$

Thus, a uniformly spaced, infinite wave train with period $\Delta$ is stable (within the kinematic approximation) if and only if $c^{\prime}(\Delta)>0$. Hence, for the dispersion curves of section 3 it would seem to a first approximation that it is always the faster of the two periodic branches that is stable. Note that where there are bumps in the dispersion curve defining so-called supernormal wave speeds (wave speeds are faster than the corresponding speed of the large period wave) then it is only the supernormal wave of smaller period that is stable. Corresponding conclusions can also be made about subnormal waves (waves of slower speed compared to the wave of large period) on the slower branch. Since homoclinic orbits bifurcate from periodic orbits we shall also conjecture that for a pair of fast/slow solitary waves the fast one is stable and the slow one unstable. These stability results are consistent with exact traveling wave solutions for the FitzHugh-Nagumo nerve equations with a piece-wise linear nonlinearity [23] and for solitary pulses in the Hodgkin-Huxley nerve equations [27].

We now consider the stability of finite non-uniform trains, rather than the more involved case of general trains of infinite extent. This allows us to make the realistic assumption that the speed of the leading pulse is $c_{0}$ (the speed of a solitary pulse) so that we may write $T^{n}(x)=x / c_{0}+\sum_{k=1}^{n} \Delta_{n}, n=1, \ldots, N$ 
[28]. Proceeding as before we find the linear equations

$$
\frac{\mathrm{d} u^{1}}{\mathrm{~d} x}=0, \quad \frac{\mathrm{d} u^{n}}{\mathrm{~d} x}=-\frac{c^{\prime}\left(\Delta_{n}\right)}{c^{2}\left(\Delta_{n}\right)}\left[u^{n}-u^{n-1}\right], \quad n=2, \ldots, N .
$$

Thus a non-uniform train is stable if and only if $c^{\prime}\left(\Delta_{n}\right)>0$ for each $n$ (ignoring the zero eigenvalue associated with translations of the leading pulse).

\subsection{Connections to periodics}

For large periods the slope of the dispersion curve in the BR model is essentially flat and the speed of the two periodic waves approximates that of the two solitary pulses. For smaller values of the period, where one does not encounter super-normal waves the stable branch of period waves has an exponential shape, which may be fitted with an equation of the form

$$
\frac{1}{c(\Delta)}=K+A \exp (-B \Delta)
$$

for some constants $K, A$ and $B$. After a rescaling $T^{n}(x) \rightarrow B\left(T^{n}(x)-n \Delta_{r}+\right.$ $\left.x / c\left(\Delta_{r}\right)\right)$ and $x \rightarrow A B \exp \left(-B \Delta_{r}\right) x$ for some arbitrary $\Delta_{r}$ the kinematic equations become

$$
\frac{\mathrm{d} T^{n}}{\mathrm{~d} x}=\exp \left(-T^{n}(x)+T^{n-1}(x)\right)-1,
$$

where we choose $\Delta_{r}$ such that $\left[K-c^{-1}\left(\Delta_{r}\right)\right] \exp \left(B \Delta_{r}\right) / A=-1$. The general solution of this system has previously been given by Horikawa [29] and discussed within the context of models of dendritic spines by Coombes [13]. Importantly, for initial data in the form of a step change in the ISIs of the form

$$
T^{n}(0)=\left\{\begin{array}{ll}
n \Delta_{(1)} & n \leq n^{*} \\
n^{*} \Delta_{(1)}+\left(n-n^{*}\right) \Delta_{(2)} & n>n^{*}
\end{array} n^{*}=\right.\text { constant }
$$

the general solution shows that the interspike intervals $\Delta_{n}(x)=T^{n}(x)-$ $T^{n-1}(x)$ may be written as a sequence with $\Delta_{n}(x)=\Delta(\kappa x-\omega n)$, for $\omega=$ $\Delta_{(2)}-\Delta_{(1)}$ and $\kappa=\exp \left(-\Delta_{(1)}\right)-\exp \left(-\Delta_{(2)}\right)$ (assuming $\left.\Delta_{(2)}>\Delta_{(1)}\right)$ where

$$
\Delta(z)=-\log \left(\exp \left(-\Delta_{(1)}\right)+\frac{\exp \left(-\Delta_{(2)}\right)-\exp \left(-\Delta_{(1)}\right)}{1+\exp (z)}\right) .
$$




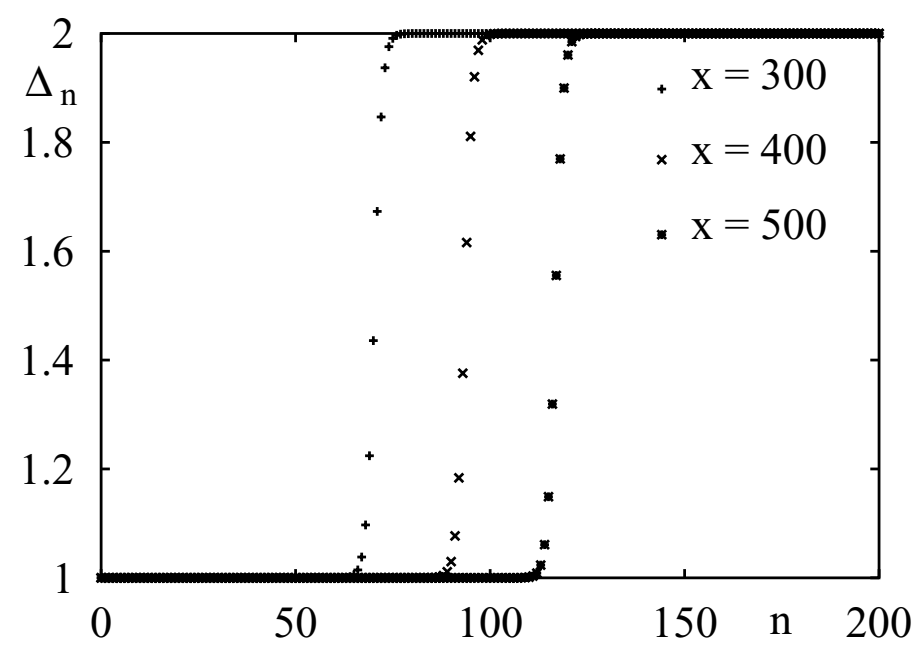

Fig. 11. A graphical illustration of the traveling front obtained analytically from the kinematic description of the SDS model. Initial data at $x=0$ is a step sequence in the interspike intervals with $\Delta_{(1)}=1$ and $\Delta_{(2)}=2$.

It is clear that $\Delta(z) \rightarrow \Delta_{(1)}$ as $z \rightarrow \infty$ and $\Delta(z) \rightarrow \Delta_{(2)}$ as $z \rightarrow-\infty$. Hence, solutions may be regarded as connections to periodic spike trains of interspike intervals $\Delta_{(1)}$ and $\Delta_{(2)}$. Moreover, the position of the front connecting the two periodic orbits moves with a constant group velocity $\mathrm{d} n / \mathrm{d} \omega=\kappa / \omega$. The front moves backwards for $\Delta_{(2)}>\Delta_{(1)}$ and forwards for $\Delta_{(1)}>\Delta_{(2)}$. In figure 11 we show a plot of the sequence of ISIs given by (14). Since the solutions describing connections between periodic orbits are constructed from a dispersion curve with $c^{\prime}(\Delta)>0$ for all realizable $\Delta$, we expect them to be stable. To check the prediction of the kinematic theory we have driven the full BR model with initial data (at one end of a cable of length $L$ ) with a spike train that has a step change in the interspike intervals.

Using the standard finite difference spatial discretization (or compartmentalization) of the system with a forward difference scheme allows a simple numerical study of the Baer and Rinzel system. For $N$ compartments (with Neumann boundary conditions) we use a spatial step size $\Delta x=L / N$ and a temporal step size of $\Delta t=1 / 4(\Delta x)^{2}$ (to satisfy the linear stability constraint). We denote here a spike train by the sequence $T^{n}(x)$ : the set of times at which the $n$th spike in the train passes at a position $x$ on the cable. At $x=0$ spike 
times are specified externally by $T^{n}(0)$, but for $x>0$ we need to be more precise about the nature of a spike. We define the time at which the $n$th spike occurs at position $x$ by introducing a threshold parameter $V_{\text {th }}$ (which we take to be $-30 \mathrm{mV}$ ) such that

$$
T^{n}(x)=\inf \left\{t \mid \widehat{V}(x, t) \geq V_{\mathrm{th}}, \frac{\partial \widehat{V}(x, t)}{\partial t}>0 ; t \geq T^{n-1}(x)\right\}
$$

Interspike intervals are defined by $\Delta_{n}(x)=T^{n}(x)-T^{n-1}(x)$. A general rectangular stimulus train has the form $I(t)=\sum_{n} P\left(t-T^{n}(0)\right)$ with $P(t)=$ $I_{0} \Theta(t) \Theta\left(\tau_{d}-t\right)$, where $I_{0}$ is the magnitude of an applied pulse, $\tau_{d}$ its duration and $\Theta(x)$ is a step function with $\Theta(x)=1$ for $x \geq 0$ and is zero otherwise. An example of such a direct simulation with $T^{n}(0)$ given by $(13)\left(I_{0}=100 \mathrm{mV}\right.$, $\tau_{d}=2 \mathrm{~ms}$ and $n^{*}=200$ ) is shown in figure 12 where it is clearly seen that the step change can smooth out to form a transition layer of the form predicted by equation (14).

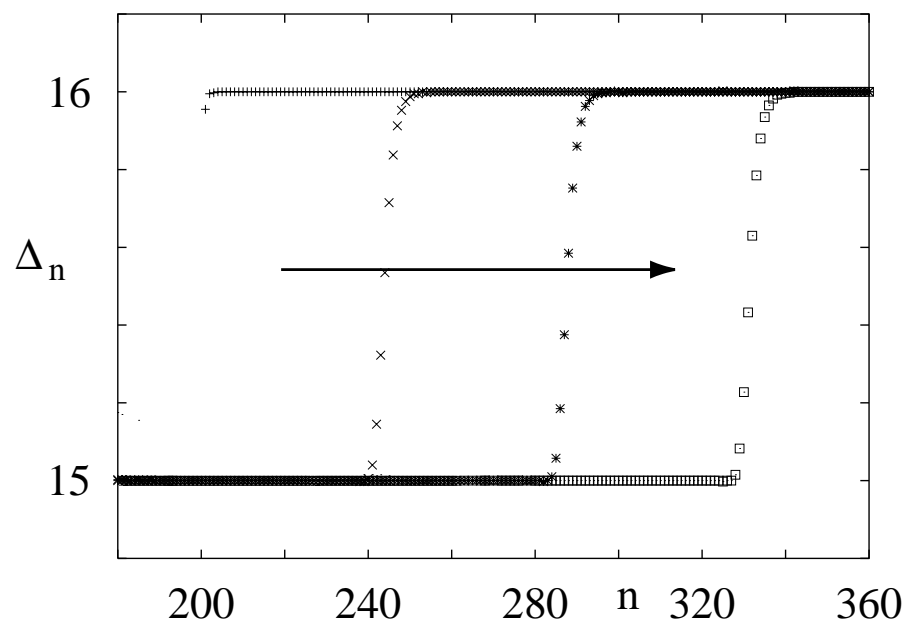

Fig. 12. An example of a connection between periodic orbits in the BR model. Initial data is in the form of a spike train with a step change in the ISIs between $\Delta_{(1)}=15$ and $\Delta_{(2)}=16$. The initial data evolves to form a smooth connection between the periodic orbits (periodic spike trains with constant ISIs $\Delta_{(1)}$ and $\Delta_{(2)}$ ) that can then propagate in an invariant fashion with constant speed. $r=1, \rho=25$, $\Delta_{(1)}=15, \Delta_{(2)}=16$. Data is shown at the following positions along the cable of length $L: 0, L / 4, L / 2$ and $3 L / 4$ (the arrow indicates the data with increasing position). 


\subsection{Bursting solutions}

From the kinematic theory, dispersion curves with stationary points (away from the minimum period) are suggestive of wave instabilities. The dispersion curve for the BR model has been shown numerically to support at least one maxima (on the faster branch), associated with waves of supernormal speed. A change in stability of periodic waves occurs when $\mathrm{d} c / \mathrm{d} \Delta=0$. From the dispersion curve of figure 8 (a) the BR model can have two different equally spaced impulse trains with the same supernormal speed. One of these is stable and the other unstable (according to the kinematic approach). Any compound trains, which are composed by the concatenations of these two solutions are also expected to be unstable [26]. Hence for a stimulus period which is larger than the average of the two supernormal wave periods (and less than the period of the unstable supernormal wave) one might expect the generation of an irregular wave train. Direct numerical simulations of the full model under these conditions show that a repetitive pulsatile stimulus can cause traveling pulses that group together to form successively larger (and less unstable) bunches. This is illustrated in figure 13 where we show the sequence of ISIs $\Delta_{n}(x)$ at a fixed distance along the cable.

\section{Discussion}

In this paper we have discussed systematic ways of describing both regular traveling waves (periodics and homoclinics) within the BR model of spine studded dendritic tissue as well irregular trains (connections to periodics and general wave trains). The former were analyzed with a numerical study of ODEs in the traveling wave frame and allowed us to describe the bifurcation sequence of regular waves in the physiologically important parameter plane comprising spine stem resistance and spine density. Both multi-pulse and periodic solutions were found to bifurcate tangentially from a solitary pulse at a co-dimension two point. The regions for propagation failure of regular waves were also constructed within this numerical framework. Importantly we also calculated the dispersion curves for periodic waves. These were then used to 


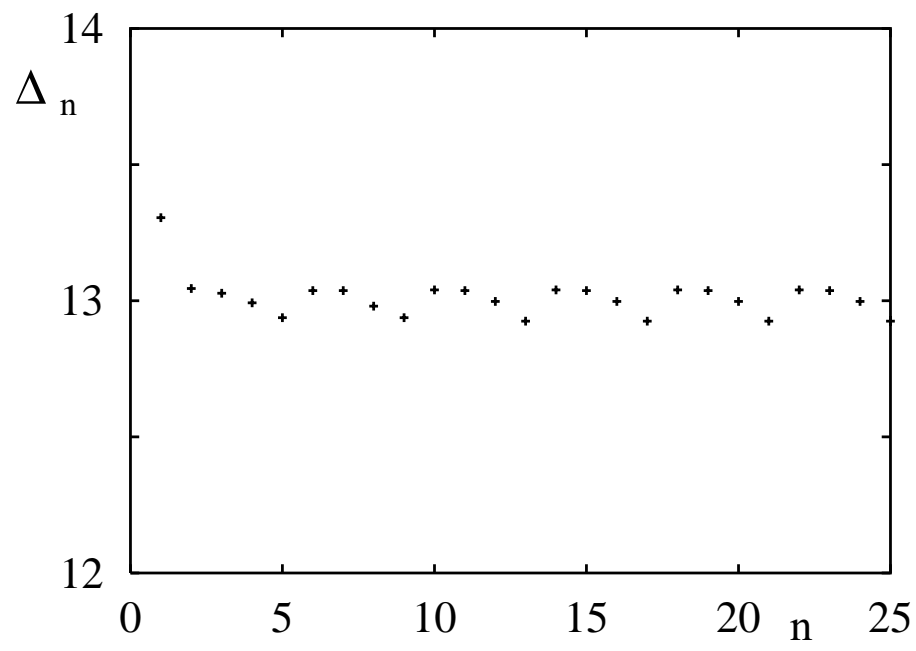

Fig. 13. An example of a bursting solution in the BR model with $r=1$ and $\rho=25$. Initial data is in the form of a periodic spike train with period $\Delta=6$. According to the kinematic theory the periodic wave of this period is unstable. The initial data evolves such that spikes tend to bunch together. Data is shown at position $L / 4$ (with numerical parameters as in figure 12).

develop a kinematic formulation of wave trains appropriate for the study of irregular wave trains and a weak form of stability for periodic and finite wave trains. A similar approach has recently been adopted by Or-Guil et al. in a study of spike trains in an excitable system with finite extent [30]. We have also used the kinematic theory to predict the existence of two special forms of irregular wave trains, both of which were subsequently found with direct numerical simulations. Moreover, stationary points of the dispersion curve were shown to be associated with period doubling bifurcations.

In a real neuron one might expect scattering of electrical waves from branch points where there is some impedance mismatch between parent and daughter dendrites. An increase in diameter or a decrease in excitability or a variation in spine density may lead to the blocking of a traveling wave with or without reflection. For an analytic or numerical study one must consider a branched structure that consist of finite pieces of spine studded cable with boundary conditions at branch points determined by Kirchoff's laws. Interestingly reflected waves have been suggested by Goldstein and Rall [31] and Ermentrout 
and Rinzel [32] to underly a form of neural pacemaker. With a suitable choice of dendritic geometry, say a piece of cable with a thin central region, a reflected wave may become trapped and bounce around periodically in some region of the dendritic tree. The kinematic approximation is likely to play an important role in the study of branched and inhomogeneous spine studded dendritic cable. In particular because small inhomogeneities can be viewed as perturbations that add to the interactions between pulses causing a modification of the instantaneous speed of a pulse in some wave train. The study of branching geometries, reflected waves and non-uniform distributions of spines are topics that are currently being investigated.

\section{Acknowledgements}

The authors would like to acknowledge support from the London Mathematical Society. 


\section{Appendix A}

For the Hodgkin-Huxley model of excitable nerve tissue it is common practice to write

$$
\begin{aligned}
\tau_{X}(\widehat{V}) & =\frac{1}{\alpha_{X}(\widehat{V})+\beta_{X}(\widehat{V})} \\
X_{\infty}(\widehat{V}) & =\alpha_{X}(\widehat{V}) \tau_{X}(\widehat{V})
\end{aligned}
$$

for $X \in\{m, n, h\}$ where

$$
\begin{aligned}
\alpha_{m}(\widehat{V}) & =\frac{0.1(\widehat{V}+40)}{1-\exp [-0.1(\widehat{V}+40)]} \\
\alpha_{h}(\widehat{V}) & =0.07 \exp [-0.05(\widehat{V}+65)] \\
\alpha_{n}(\widehat{V}) & =\frac{0.01(\widehat{V}+55)}{1-\exp [-0.1(\widehat{V}+55)]} \\
\beta_{m}(\widehat{V}) & =4.0 \exp [-0.0556(\widehat{V}+65)] \\
\beta_{h}(\widehat{V}) & =\frac{1}{1+\exp [-0.1(\widehat{V}+35)]} \\
\beta_{n}(\widehat{V}) & =0.125 \exp [-0.0125(\widehat{V}+65)]
\end{aligned}
$$

All potentials are measured in $\mathrm{mV}$, all times in $\mathrm{ms}$ and all currents in $\mu \mathrm{A}$ per $\mathrm{cm}^{2}$. We use the following parameter values: $\widehat{C}=1 \mu \mathrm{F} \mathrm{cm} \mathrm{cm}^{-2}, g_{L}=0.3$, $g_{K}=36, g_{N a}=120, V_{L}=-54.402, V_{K}=-77$ and $V_{N a}=50$.

\section{References}

[1] K M Harris and S B Kater. Dendritic spines: cellular specializations imparting both stability and flexibility to synaptic function. Annual Review of Neuroscience, 17:341-371, 1994.

[2] R Yuste and W Denk. Dendritic spines as basic functional units of neuronal integration. Nature, 375:682-684, 1995.

[3] K M Hariss. Calcium from internal stores modifies dendritic spine shape. Proceedings of the National Academy of Sciences USA, 96:12213-12215, 1999. 
[4] G M Shepherd and R K Brayton. Logic operations are properties of computersimulated interactions between excitable dendritic spines. Neuroscience, 21(1):151-165, 1987.

[5] B W Mel. NMDA-based pattern discrimination in a modeled cortical neuron. Neural Computation, 4:502-517, 1992.

[6] K T Blackwell, T P Vogl, and D L Alkon. Pattern matching in a model of dendritic spines. Network, 9:107-121, 1998.

[7] I Segev and W Rall. Excitable dendrites and spines: earlier theoretical insights elucidate recent direct observations. Trends in Neuroscience, 21(11):453-460, 1998.

[8] S M Baer and J Rinzel. Propagation of dendritic spines mediated by excitable spines: A continuum theory. Journal of Neurophysiology, 65(4):874-890, 1991.

[9] P Chen and J Bell. Spine-density dependence of the qualitative behaviour of a model of a nerve fiber with excitable spines. Journal of Mathematical Analysis and Applications, 187:384-410, 1994.

[10] Y Zhou and J Bell. Study of propagation along nonuniform excitable fibers. Mathematical Biosciences, 119:169-203, 1994.

[11] Y Zhou. Unique wave front for dendritic spines with Nagumo dynamics. Mathematical Biosciences, 148:205-225, 1998.

[12] S Coombes and P C Bressloff. Solitary waves in a model of dendritic cable with active spines. SIAM Journal on Applied Maths, 61:432-453, 2000.

[13] S Coombes. From periodic travelling waves to travelling fronts in the spikediffuse-spike model of dendritic waves. Mathematical Biosciences, 170:155-172, 2001.

[14] J Cronin. Mathematical aspects of Hodgkin-Huxley neural theory. Cambridge University Press, 1987.

[15] W J Beyn. The numerical computation of connecting orbits in dynamical systems. IMA Journal of Numerical Analysis, 9:379-405, 1990.

[16] E J Doedel, A R Champneys, T R Fairgrieve, Y A Kuznetsov, B Sandstede, and X J Wang. AUTO97 continuation and bifurcation software for ordinary differential equations. 1997. Available by anonymous ftp from FTP.CS.CONCORDIA.CA, directory PUB/DOEDEL/AUTO. 
[17] B Sandstede. Convergence estimates for the numerical approximation of homoclinic solutions. IMA Journal of Numerical Analysis, 17:437-462, 1997.

[18] B Sandstede. Stability of multiple-pulse solutions. Transactions of the American Mathematical Society, 350:429-472, 1998.

[19] R N Miller and J Rinzel. The dependence of impulse propagation speed on firing frequency, dispersion, for the Hodgkin-Huxley model. Biophysical Journal, 34:227-259, 1981.

[20] P Glendinning. Travelling wave solutions near isolated double-pulse solitary waves of nerve axon equations. Physics Letters A, 121:411-413, 1987.

[21] L P Šilnokov. A case of the existence of a denumerable set of periodic motions. Sov. Math. Dokl., pages 163-166, 1965.

[22] J A Feroe. Traveling waves of ininitely many pulses in nerve equations. Mathematical Biosciences, 55:189-203, 1981.

[23] J Rinzel and J B Keller. Traveling wave solutions of a nerve conduction equation. Biophysical Journal, 13:1313-1337, 1973.

[24] G A Carpenter. A geometric approach to singular perturbation problems with applications to nerve impulse equations. Journal of Differential Equations, 23:335-367, 1977.

[25] B E Oldeman, B Krauskopf, and A R Champneys. Death of period-doublings: locating the homoclinic-doubling cascade. Physica D, 146:100-120, 2000.

[26] J Rinzel and K Maginu. Kinematic analysis of wave pattern formation in excitable media. In C Vidal and A Pacault, editors, Non-Equilibrium Dynamics in Chemical systems, pages 107-113. Springer-Verlag, 1984.

[27] J W Evans and J Feroe. Local stability theory of the nerve impulse. Mathematical Biosciences, 37:23-50, 1977.

[28] C Elphick, E Meron, J Rinzel, and E A Spiegel. Impulse patterning and relaxational propagation in excitable media. Journal of Theoretical Biology, 146:249-268, 1990.

[29] Y Horikawa. A spike train with a step change in the interspike intervals in the FitzHugh-Nagumo model. Physica D, 82:365-370, 1995. 
[30] M Or-Guil, I G Kevrekidis, and M Bär. Stable bound states of pulses in an excitable medium. Physica D, 135:154-174, 2000.

[31] S S Goldstein and W Rall. Changes of action potential shape and velocity for changing core conductor geometry. Biophysical Journal, 14:731-757, 1974.

[32] G B Ermentrout and J Rinzel. Reflected waves in an inhomogeneous excitable medium. SIAM Journal on Applied Mathematics, 56:1107-1128, 1996. 